\title{
Solar cycle activity: A preliminary prediction for cycle \#24
}

\author{
S. Sello ${ }^{\star}$ \\ Mathematical and Physical Models, Enel Research, via Andrea Pisano 120, 56122 Pisa, Italy \\ Received 16 June 2003 / Accepted 30 July 2003

\begin{abstract}
Solar activity forecasting is an important topic for numerous scientific and technological areas, such as space operations, electric power transmission lines and earth environment impact. Nevertheless, the well-known difficulty is how to accurately predict, on the basis of various recorded solar activity indices, the complete evolution of future solar cycles, due to highly complex dynamical processed involved, mainly related to interaction of different components of internal magnetic fields. There are mainly two distinct classes of solar cycle prediction methods: the precursor-like ones and the mathematicalnumerical ones. The main characteristic of precursor techniques, both purely solar and geomagnetic, is their physical basis. The non-precursor methods use different mathematical properties of the known temporal evolution of solar activity indices to extract information useful for predicting future activity. For the current solar cycle \#23 we obtained quite good performances from both some precursor and purely numerical methods, such as the so-called solar precursor and nonlinear ones. To further check the performances of these prediction techniques, we compared the early predictions for the next solar cycle \#24. Preliminary results support the coherence of the prediction methods considered and confirm the actual trend of a reducing solar activity.
\end{abstract}

Key words. Sun: activity - Sun: magnetic fields

\section{Introduction}

The current solar cycle \#23 is in its declining phase following a multipeaked maximum around April 2000 (principal peak: $R_{z}=120.8$ ) and November 2001 (secondary peak: $R_{z}=115.5$ ), for the monthly smoothed sunspot numbers (data from SIDC 2003). Many solar cycle forecasting methods predicted higher values, including statistical, curve fitting, spectral one and some geomagnetic precursor-like ones. A notable exception in the class of precursors, was the pure solar precursor method developed by Schatten and co-workers, which adequately predicted, using the SODA index, a lower value: $R_{z}=138 \pm 30$ (Schatten \& Pesnell 1993). In the class of numerical based methods, we found a good global forecast performance obtained by the classical McNish-Lincoln method, modified using regression coefficients and mean cycle values computed for Cycles \#8 through \#20, followed by a nonlinear method based on the chaos theory (Sello 2001). In the evaluation we used a global error estimation based on the whole shape of the curve prediction, not only the maximum peak amplitude and related phase. Although the actual McNish-Lincoln method was designed for short-term predictions (about one year in advance), this modified version has given quite good mediumterm results, perhaps due to a relatively slow increase phase characterizing the current solar activity cycle, (Sello 2001). An important feature of the current solar cycle is the violation of the Odd/Even hypothesis where odd-numbered cycles have greater activity than the preceding even-numbered ones

\footnotetext{
* e-mail: stefano.sello@enel.it
}

(Gnevyshev-Ohl rule). Another notable characteristic is the pronounced double maximum, a complex structure very difficult to predict in advance, even using the most refined prediction methods (Sello 2003). At the present phase of solar cycle evolution, it is too early to accurately predict the next cycle development, peak amplitude and phase, for every solar cycle prediction method. Nevertheless, there are already different preliminary attempts to estimate the future solar cycle amplitudes and timings.

Here we focus attention on a recent work by Schatten (Schatten 2002), which uses the refined concepts of the solar precursor method to give an early estimate of the next solar cycle amplitude and phase. For a comparison with a nonprecursor technique, we use also the nonlinear method described in Sello (2001).

\section{Solar precursor method}

Among the class of solar cycle prediction methods, the socalled precursor ones are mainly based on physical concepts, directly related to solar magnetic processes. In particular, the solar precursor method, developed by Schatten \& Pesnell (1993), appears as the best founded approach since it uses only the information about the Sun's polar magnetic field as a precursor to the future intensity of solar activity. More precisely, using the concepts of the solar dynamo mechanism, the method assumes that the polar magnetic field in the descending and minimum phases of a given cycle, produces information on the future fully developed toroidal fields inside the Sun that 
drive the next solar activity cycle. To extend the usual precursor methods and to enforce their physical basis, the authors attempted to evaluate the amount of hidden dynamo magnetic flux within the solar interior during any phase of the solar cycle using a proper index, called SODA (for Solar Dynamo Amplitude). The initial formulation of the SODA index was the following: (Schatten \& Pesnell 1993)

$\operatorname{SODA}(t)=60+146\left\{\left[\frac{F 10.7(t)-60}{146}\right]^{2}+\left[\frac{B_{\text {polar }}(t)}{128}\right]^{2}\right\}^{1 / 2}$

where: $10.7(t)$ is the radio emission from the Sun at a wavelength of $10.7 \mathrm{~cm}$ which has been found to correlate quite well with sunspot numbers; $B_{\text {polar }}(t)$ is the polar field strength at time $t$. These quantities can be assumed as proxies to estimate the intensity of the toroidal and poloidal solar magnetic field components respectively. A direct estimation of polar and toroidal components at a given time allow us to evaluate the predicted values by using the SODA index to obtain $F 10.7$ flux from the latest smoothed values. The radio flux is then converted to sunspot numbers through a relation based on correlation. Based on good predicted values for current solar cycle \#23, Schatten refines the method improving the cycle timing aspect of the prediction technique using both the equatorward march of active regions and the reversal of the Sun's polar fields (Schatten 2002).

\section{Nonlinear dynamics method}

The intrinsic complexity in the temporal behavior of sunspot numbers (irregular and intermittent) and other solar activity indices suggested the possibility of a nonlinear (sometimes chaotic) dynamics governing the related physical processes. In a previous work we have shown the efficiency of a numerical method based on nonlinear dynamics techniques, properly designed to predict the medium-time evolution of the current solar cycle \#23 (Sello 2001). In particular, the prediction model is based on the assumption that the underlying dynamics, driven by the evolution of magnetic fields during a solar cycle, is well described by a nonlinear deterministic behavior in a proper embedding space, i.e. a space that mimics the original phase space, (Abarbanel et al. 1990):

$$
f^{T}\left(\boldsymbol{y}_{t}\right)=\boldsymbol{y}_{t+T}
$$

for a given embedding vector: $\boldsymbol{y}$. The inverse problem to be solved consists of the computation of the smooth map $f^{T}$, given a scalar time series (here the sunspot numbers). Following the approach given by Zhang (1996), we designed a modified improved version that uses different tools of nonlinear dynamics theory, such as mutual information, linear and nonlinear redundancy, Lyapunov's spectrum etc. Describing the dynamic evolution in a 5-dimensional embedding space, the predicted behavior of the ascending phase, maximum peak value $\left(R_{z}=125.6 \pm 7.1\right)$, its timing (April-May 2000) and the early descending phase, for the current solar cycle \#23, was quite accurate (Sello 2001). Moreover, the analysis clearly pointed out the nonlinear-chaotic nature, with limited predictability (4.8 years), of the solar cycle activity. The main

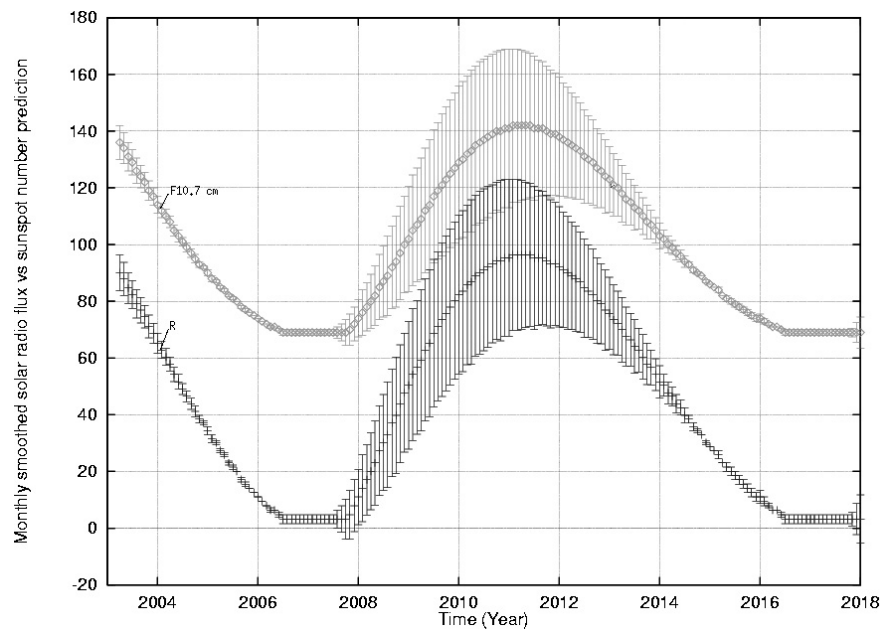

Fig. 1. Predicted descending phase of the current solar cycle \#23 and the next solar cycle \#24: $10.7 \mathrm{~cm}$ radio flux with uncertainty $\pm 1 \sigma$ from Schatten (2002) and the related monthly smoothed sunspot numbers $\mathrm{R}$.

difficulty is to include in the model some high amplitude stochastic components of solar activity. Another difficulty seems to be the prediction of the specific multipeaked shape near the maximum phase. In the descending phase of the current solar cycle, an application of the nonlinear prediction method can be quite useful, in order to obtain an early estimate of the next solar cycle \#24's behavior. This allows us a preliminary comparison between the (non-precursor) nonlinear method and the solar precursor one.

\section{An early prediction of cycle \#24}

The prediction of the next solar cycle given by Schatten is based on the SODA index, as well described in detail in a recent work (Schatten 2002). Starting from the toroidal and poloidal fields proxy data the author obtained a value for the next cycle peak coronal activity index (a proxy for $F 10.7$ flux) near $175 \pm 50$ peaking in April $2011 \pm 3$ months. To follow in more detail the updated predicted monthly mean smoothed estimates of 10.7 solar radio flux we plotted the complete values with their uncertainty from the GSFC (NASA) database (March 2003, nominal timing) (Schatten 2002, 2003) (Fig. 1). The conversion from the radio flux values to monthly averaged sunspot numbers uses an equation derived by correlation, which is valid on a statistical basis (Thompson 2003):

$$
R_{z}(t)=1.61 F D(t)-(0.0733 F D(t))^{2}+(0.0240 F D(t))^{3}
$$

where: $F D(t)=F 10.7(t)-67.0$.

From these curves we derived that the predicted peak amplitude for the monthly smoothed sunspot numbers in the next solar cycle \#24 is near $96 \pm 25$, peaking in April 2011. This prediction confirms the current trend towards reduced solar activity cycles.

In Fig. 2 we show the comparison between the solar precursor and the nonlinear predictions for the monthly smoothed sunspot numbers for the descending part of the current solar cycle and for all of the next cycle. 


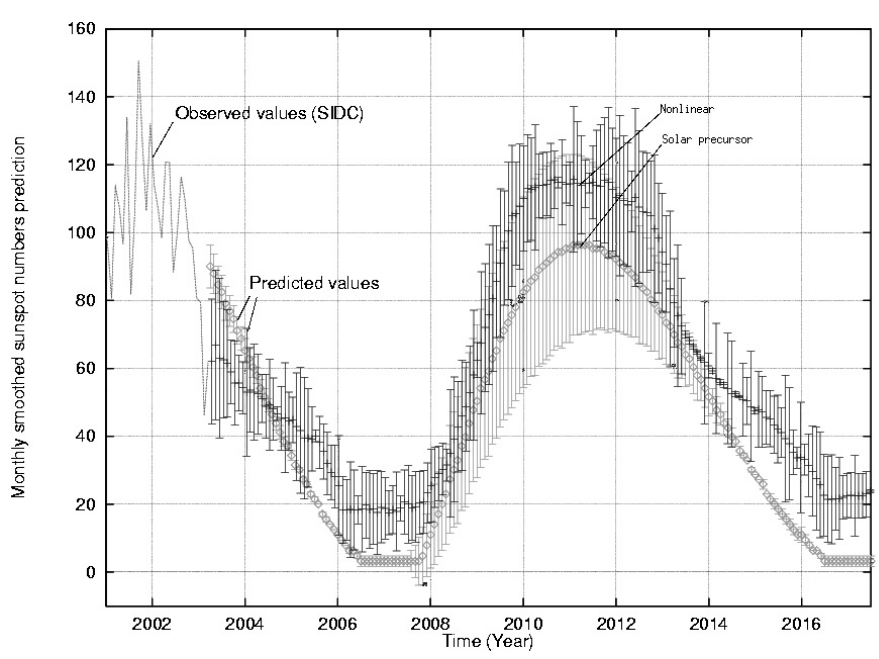

Fig. 2. Predicted descending phase of the current solar cycle \#23 and the next solar cycle \#24 for monthly smoothed sunspot numbers: comparison between solar precursor (square symbols) and nonlinear prediction (cross symbols) methods. The plot includes the observed sunspot numbers updated to March 2003 (from: SIDC, RWC Belgium, World Data Center for the Sunspot Index, Royal Observatory of Belgium 2003).

Taking into account the uncertainties, the nonlinear prediction method appears very coherent, both in amplitude and in phase, with the solar precursor one, estimating a mean peak value slightly broader and higher: $115 \pm 21$, peaking in the year 2011. We note that the use of the nonlinear method for time intervals greater than the sunspot numbers Lyapunov's time (error doubling predictability time), here 4.8 years, needs to be reiterated, using previously predicted values, to cover a complete solar cycle (Sello 2001). These early predictions, for the next solar cycle \#24, support the tendency towards a reduction in the intensity of solar cycle activity. Another early prediction of the maximum sunspot number in solar cycle \#24 was recently given by Duhau (2003). In this work a suitable nonlinear coupling function between long-term variations in the geomagnetic index aa and sunspot numbers was derived by a wavelet analysis to separate three main time scales: Schwabe, decadal and secular ones. In this case the modulations of aa-minima and $R$-maxima are seen as measures of the polar and toroidal magnetic field intensities respectively. Assuming the actual solar dynamo to be a quasi-harmonic process, the author found, for the maximum yearly mean sunspot number in solar cycle $\# 24$, the value $87 \pm 23.5$ in agreement with the above predictions. Further, the Gnevyshev-Ohl rule would probably be violated for two consecutive odd-even cycle pairs.

\section{Conclusions}

The predictions of the current 23rd solar cycle activity show that the complexity of the solar processes related to the evolution of magnetic fields prevents any accurate forecasting of the solar cycle curve shape and in particular an accurate estimation for both amplitude and phase of future cycles. Nevertheless, different methods, both precursor and non-precursor, have obtained quite good overall performances, suggesting the reliability and usefulness of these prediction approaches. To further check the performances of some prediction techniques, we compared the early predictions, for the next solar cycle \#24, of a solar precursor method and a nonlinear numerical one. The preliminary results support the reliability and coherence of the prediction techniques considered: for the next solar cycle we confirm the actual trend of reduced solar activity and violation of the Gnevyshev-Ohl rule.

\section{References}

Abarbanel, H. D. I., Brown, R., \& Kadtke, J. B. 1990 Phys. Rev. A, 41,4

Duhau, S. 2003, Sol. Phys., 213, 1, 203

GSFC (NASA) 2003 http: //fdf.gsfc.nasa.gov/dist/ generalProducts/database/

Schatten, K. H., \& Pesnell, W. D. 1993, Geophys. Res. Lett., 20, 2275

Schatten, K. H. 2002, J. Geophys. Res., 107, A11, SSH15

Sello, S. 2001, A\&A, 377, 1, 312

Sello, S. 2003, New Astron., 8, 2, 105

SIDC 2003, Solar Influences Data Analysis Center, Brussels http://www . oma.be/

Thompson, R. IPS Radio and Space Services 2003, http://www.ips.gov.au

Zhang, Q. 1996, A\&A, 310, 646 